\title{
Low-Temperature Growth of InGaN Films over the Entire Composition Range by MBE
}

\author{
Chloe A. M. Fabien ${ }^{\mathrm{a}}$, Brendan P. Gunning ${ }^{\mathrm{a}}$, W. Alan Doolittle ${ }^{\mathrm{a}^{*}}$ \\ Alec M. Fischer ${ }^{b}$, Yong O. Wei ${ }^{b}$, Hongen Xie ${ }^{b}$, and Fernando A. Ponce \\ ${ }^{a}$ School of Electrical and Computer Engineering, Georgia Institute of Technology, Atlanta, GA 30332, \\ USA. \\ ${ }^{\mathrm{b}}$ Department of Physics, Arizona State University, Tempe, AZ 85281, USA. \\ * Corresponding author at: Georgia Institute of Technology, Atlanta, GA 30332, USA. \\ Tel/ Fax: +1 4048949884 \\ E-mail address: alan.doolittle@ece.gatech.edu (W. A. Doolittle)
}

Keywords: B1 Nitrides; A3 Molecular beam epitaxy; A1 Crystal structure; A1 X-ray diffraction; A1 Atomic force microscopy

\begin{abstract}
The surface morphology, microstructural, and optical properties of indium gallium nitride (InGaN) films grown by plasma-assisted molecular beam epitaxy under low growth temperatures and slightly nitrogen-rich growth conditions are studied. The single-phase InGaN films exhibit improved defect density, an absence of stacking faults, efficient In incorporation, enhanced optical properties, but a grainlike morphology. With increasing In content, we observe an increase in the degree of relaxation and a complete misfit strain relaxation through the formation of a uniform array of misfit dislocation at the InGaN/GaN interface for InGaN films with indium contents higher than 55-60\%.
\end{abstract}

\section{Introduction}

The ternary indium gallium nitride ( $\mathrm{InGaN})$ alloy system is of great interest for optoelectronics applications due to its direct band gap varying from 0.65 to $3.42 \mathrm{eV}$ and thus, spanning the entire visible to near-infrared spectrum [1]. However, the epitaxial growth of InGaN over the entire composition range faces many issues such as (i) the lack of appropriate substrates, which leads to dislocation formation [2], (ii) the control of In incorporation, which depends on the growth chemistry and temperature [3], and (iii) the miscibility gap between $\mathrm{InN}$ and $\mathrm{GaN}$, which results in phase separation [4]. 
A precise control and optimization of the III/N ratio and the growth temperature is required to obtain single-phase, high-quality InGaN films. The growth of single-phase InGaN by molecular beam epitaxy (MBE) has been previously reported using various approaches [5-8]. Metal-rich growth favors a 2D growth mode, which promotes smoother surfaces but results in compositional alloy fluctuations and high threading dislocation densities $[5,6]$. On the other hand, N-rich growth leads to more uniform InGaN alloys but reduces surface adatom mobility, resulting in 3D growth mode and grain-like morphology [68]. The structural and optical properties of InGaN films also strongly depend on growth temperature [9]. An optimized growth temperature must account for a tradeoff between enhanced compositional homogeneity and In incorporation at low temperatures and improved surface morphology and threading dislocation density at high temperatures.

In order to take advantage of both metal-rich and N-rich conditions, several modulated growth methods have been studied [10, 11]. Metal-modulated epitaxy (MME), a growth technique that uses constant $\mathrm{N}$ flux, shuttered Ga and In fluxes under metal-rich conditions, and extremely low substrate temperatures $\left(400-450^{\circ} \mathrm{C}\right)$, has enabled the growth of InGaN films throughout the miscibility gap [12, 13]. While the moderate In-content films (22-46\% $\mathrm{InGaN})$ display high density of dislocations and stacking faults, InGaN films with In content higher than $60 \%$ exhibit a significant improvement in the crystalline quality and optical properties due to a complete misfit strain relaxation through the formation of a uniform array of misfit dislocations at the InGaN/GaN interface [14]. However, it is unclear whether this complete misfit strain relaxation originates from (i) the fundamental lattice mismatch between In-rich InGaN films and GaN, (ii) the MME growth technique, or (iii) the use of low temperatures. In this work, we report on the growth of InGaN films over the entire composition range under very low growth temperatures and slightly N-rich growth conditions.

\section{Experiments}

The InGaN films were grown in a Riber 32 MBE system using a standard effusion cell for In, a Veeco SUMO cell for Ga, and a Veeco Unibulb plasma source for N. Lumilog MOCVD-grown GaN (0001) 
templates on sapphire were used as substrates and were backside metallized with tantalum to enable radiative heating. Substrates were degreased in $\mathrm{H}_{2} \mathrm{SO}_{4}: \mathrm{H}_{2} \mathrm{O}_{2}$ (4:1) and outgassed in the introductory chamber at $150^{\circ} \mathrm{C}$ for $30 \mathrm{~min}$. A $\sim 130 \mathrm{~nm} \mathrm{GaN}$ buffer was grown at $600^{\circ} \mathrm{C}$ via MME to bury the native surface oxide.

InGaN growth was performed at various low growth temperatures $\left(360-450^{\circ} \mathrm{C}\right)$ depending on the targeted compositions. All InGaN films were grown under N-rich conditions using a constant III/N ratio of $\sim 0.9$ with group-III fluxes normalized by Z-number to account for differences in ion-gauge sensitivity between In and Ga. The In/(Ga+In) ratio was varied to obtain InGaN alloys over the entire composition range. Nitrogen flow rate and RF-plasma power were set at $1.3 \mathrm{sccm}$ and $350 \mathrm{~W}$, respectively. The growth rate was $\sim 1 \mu \mathrm{m} / \mathrm{hr}$ and the nominal $\mathrm{InGaN}$ film thickness was $55 \mathrm{~nm}$. The InGaN layers were then capped with a $\sim 10 \mathrm{~nm}$ GaN film to prevent thermal decomposition of the surface upon cool down.

The growth of the InGaN films was monitored in situ by reflection high-energy electron diffraction (RHEED). The structural properties of the samples were characterized by X-ray diffraction (XRD)

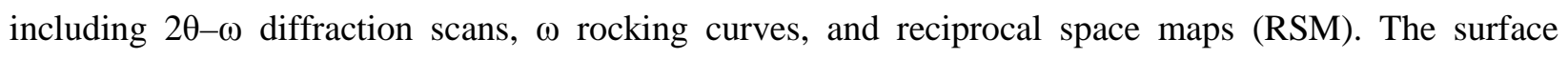
morphology, microstructural, and optical properties of all these samples were analyzed by atomic force microscopy (AFM), transmission electron microscopy (TEM), and photoluminescence/cathodoluminescence (PL/CL), respectively.

\section{Results and discussion}

Fig. 1 shows the In content determined by XRD as a function the ratio of the indium (In) to the total group-III (In + Ga) flux. The experimental data points follow a linear behavior with a slope equal to one. This slope can be interpreted as the incorporation efficiency of In into the InGaN layer, which depends on the desorption and the segregation of In atoms $[8,15]$. At the low substrate temperatures used for these growths, desorption of In atoms from the surface is negligible. Under N-rich growth, the influence of In segregation is limited due to the reduced surface adatom mobility. Therefore, at low substrate temperatures and under slightly $\mathrm{N}$-rich conditions, the In incorporation efficiency is approximately unity. 


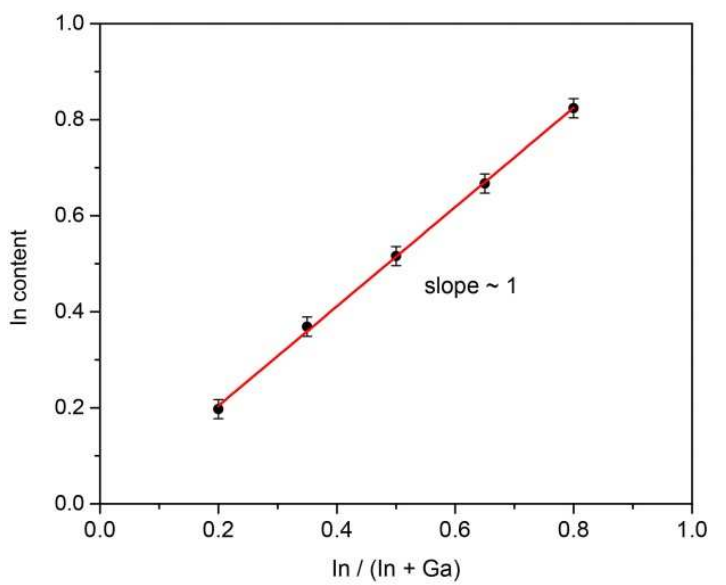

Figure 1 In content of InGaN films as a function of the ratio of In to total metal flux. The slope represents the In incorporation efficiency.

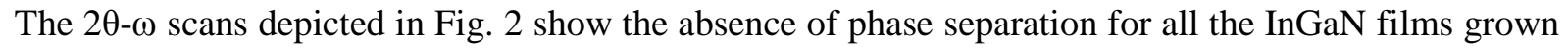
throughout the miscibility gap. The suppression of phase separation can be attributed to kineticallylimited adatom diffusion enhanced by the N-rich growth regime [6] and the low substrate temperatures [9]. The (0002) rocking curve FWHMs of all InGaN films are comparable to those of the underlying GaN templates, indicating negligible tilt. Film thicknesses were measured to be $52-60 \mathrm{~nm}$ from the Pendellösung fringes, which are also indicative of smooth interfaces. The surface morphology of the InGaN films was investigated by AFM. The AFM images shown in Fig. 3 display smooth surfaces with sub-nm RMS roughness. The low adatom mobility from the slightly N-rich growth conditions results in a combination of long-range 2D atomic steps (Fig. 3a) with short-range 3D granular domains (Fig. 3c) that are $25-50 \mathrm{~nm}$ diameter wide. This grainy morphology is confirmed by the slightly spotty RHEED pattern observed during growth as shown in Fig. 3d.

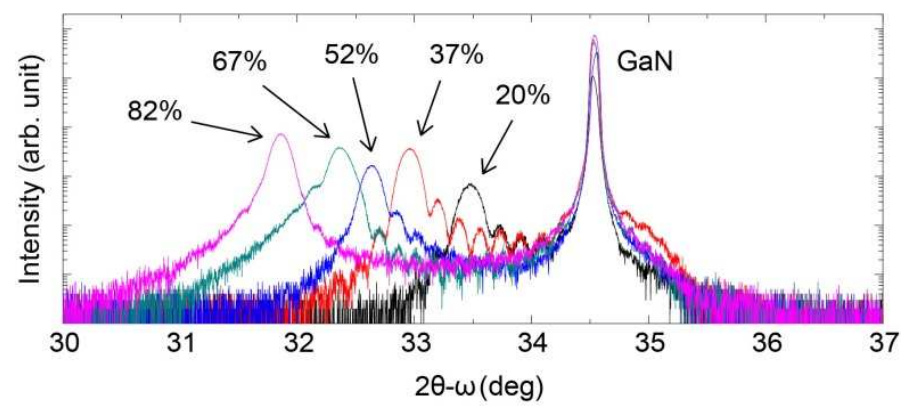

Figure $22 \theta-\omega$ diffraction scans of single-phase InGaN films over the entire composition range. 

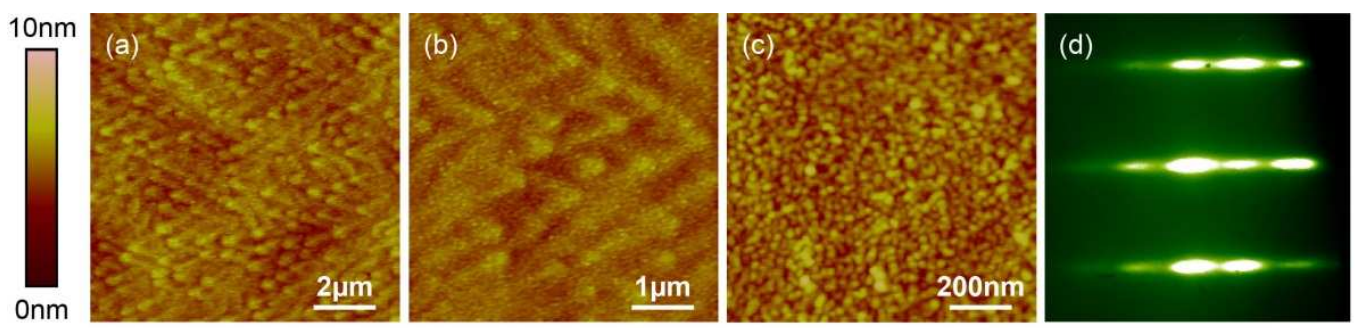

Figure 3 (a) $10 \times 10 \mu \mathrm{m}$, (b) $5 \times 5 \mu \mathrm{m}$, and (c) $1 \times 1 \mu \mathrm{m}$ representative AFM images of InGaN films grown under slightly N-rich conditions at low temperatures. (d) Typical RHEED pattern during growth.

The composition and strain of the InGaN films were derived from XRD reciprocal space maps. Fig. 4 depicts the InGaN/GaN RSMs for the (1015) reflections of the five InGaN samples with In compositions ranging from $20 \%$ to $82 \%$. The fully-strained and fully-relaxed lines are also represented as vertical and diagonal dashed lines, respectively. With increasing In content, the InGaN reciprocal lattice point shifts from a fully-strained to a fully-relaxed position. The $20 \%$ InGaN film is fully coherent to the GaN underlayer. The degree of relaxation increases for the $37 \%$ and the $52 \%$ InGaN films and for In composition of $67 \%$ and $82 \%$, the films are fully relaxed. This relaxation trend is confirmed by TEM as shown in Fig. 5. The 20\% InGaN film exhibits residual strain. The 37\% InGaN layer follows an islandnucleation growth mechanism, resulting in isolated islands, with different tilt and twist, which coalesce. The onset of moiré fringes, which are associated with misfit dislocations, is observed at the GaN/InGaN interface for the $52 \%$ InGaN as demonstrated by the periodic peaks from the line scan (Fig. 5c inset). The $\mathrm{N}$-rich $67 \%$ InGaN sample clearly exhibits moiré fringes at the interface, indicating a complete misfit strain relaxation through misfit dislocations. Similar to the MME-grown InGaN samples [14], we observe a transition in the structural properties of the N-rich InGaN films for In content higher than 55\%-60\%. This transition coincides with the calculated critical thickness reaching a value close to a monolayer. At this composition, due to the large lattice mismatch between $\mathrm{GaN}$ and In-rich InGaN, the material spontaneously nucleates in a fully relaxed state, with the formation of a uniform array of misfit dislocations at the interface. 


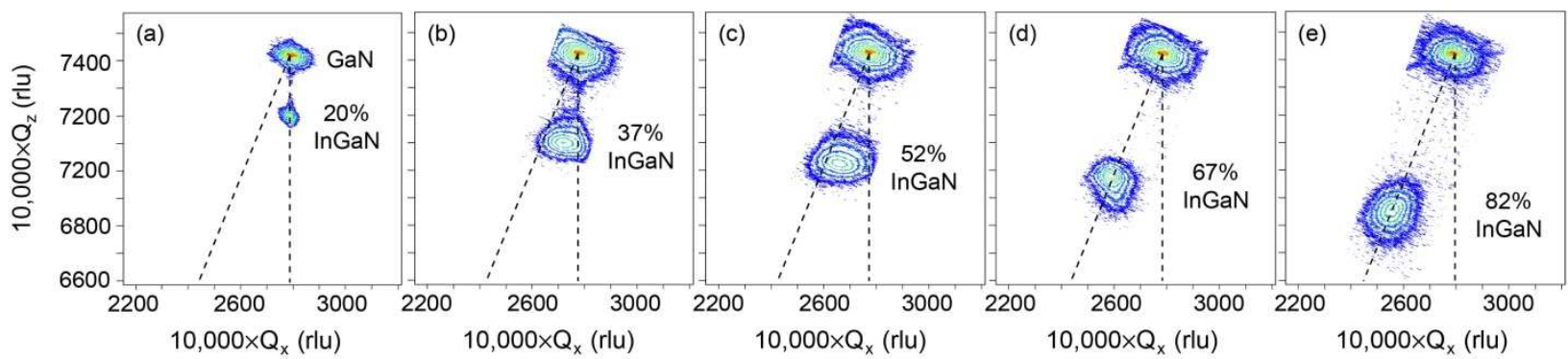

Figure 4 Reciprocal space maps along the (1015) reflection of the (a) $20 \%$, (b) $37 \%$, (c) $52 \%$, (d) $67 \%$, and (e) $82 \%$ InGaN films. The vertical and diagonal dashed lines corresponds to the fully-strained and fully-relaxed positions, respectively.
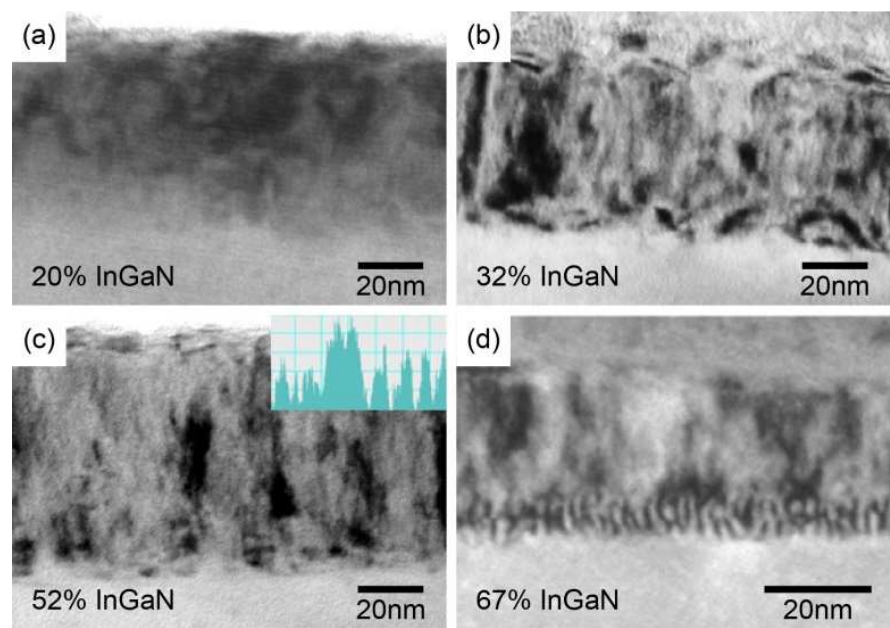

Figure 5 Cross-section TEM images of the InGaN films, taken along the $\langle 11 \overline{2} 0\rangle$ projection with diffraction contrast set at $\mathrm{g}=[1 \overline{1} 00]$. Residual strain and island formation are observed for the (a) $20 \%$ and (b) 37\% InGaN films, respectively. Moiré fringes indicating misfit strain relaxation are observed at the InGaN/GaN interfaces for the (c) $52 \%$ and (d) $67 \%$ InGaN films. The inset shows periodic peaks from the line scan at the bottom $\mathrm{InGaN} / \mathrm{GaN}$ interface.

All these InGaN films exhibit luminescence either by CL (Fig. 6a) or PL (Fig. 6b) possibly due to reduced defect density compared to the MME-grown InGaN films [14] and the absence of stacking faults as demonstrated by the TEM. The additional peaks and shoulders in the $67 \%$ and $82 \%$ InGaN spectra are attributed to Fabry-Pérot interference from the GaN underlayer. 
(a)

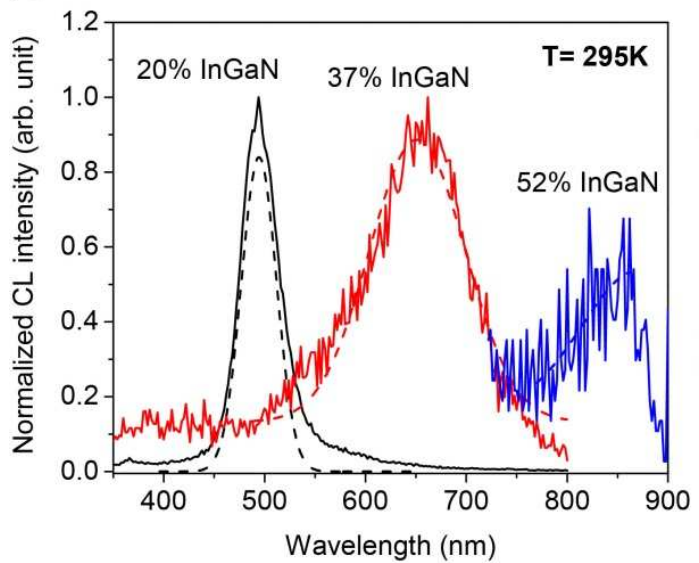

(b)

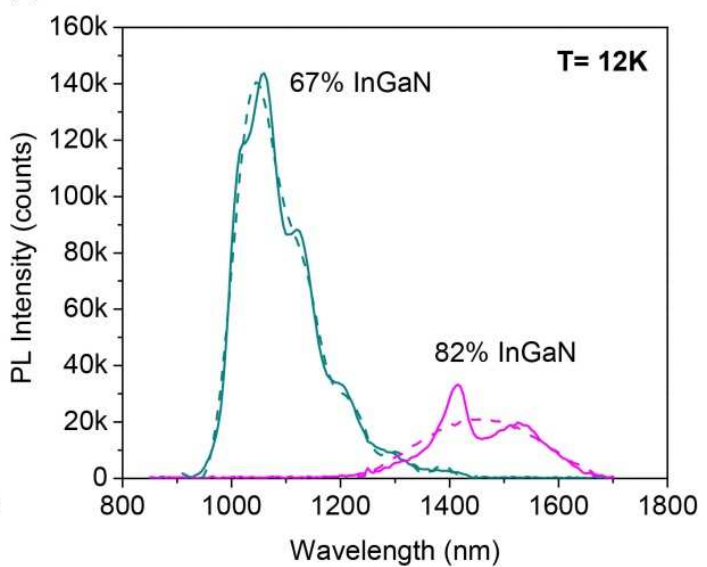

Figure 6 (a) Cathodoluminescence spectra of the 20\%, 37\%, and 52\% InGaN films and (b) photoluminescence spectra of the $67 \%$ and $82 \%$ InGaN films. The dashed lines represent the Gaussian fittings after removal of Fabry-Pérot interference effects.

\section{Conclusions}

In summary, single-phase InGaN films over the entire composition range have been grown by plasmaassisted MBE under slightly N-rich growth regime at extremely low temperatures. These growth conditions resulted in low defect density, notably no observation of stacking faults, efficient In incorporation, the suppression of phase separation, an improvement in the optical properties, and a grainy surface morphology. We observed an increase in the relaxation degree with higher In content and a transition in the structural properties of the InGaN films for In content higher than $60 \%$.

\section{Acknowledgement}

This material is based upon work supported in part by the National Science Foundation (NSF) and the Department of Energy (DOE) under NSF CA No. EEC-1041895. Any opinions, findings and conclusions or recommendations expressed in this material are those of the author(s) and do not necessarily reflect those of NSF or DOE. The information, data, or work presented herein was funded in part by the Advanced Research Projects Agency-Energy (ARPA-E), U.S. Department of Energy, under Award Number DE-AR0000470. 


\section{References}

[1] J. Wu, When group-III nitrides go infrared: New properties and perspectives, J. Appl. Phys. 106 (2009) 011101.

[2] L. Liu, J.H. Edgar, Substrates for gallium nitride epitaxy, Mater. Sci. Eng., R 37 (2002) 61-127.

[3] F.K. Yam, Z. Hassan, InGaN: An overview of the growth kinetics, physical properties and emission mechanisms, Superlattices Microstruct. 43 (2008) 1-23.

[4] R. Singh, D. Doppalapudi, T.D. Moustakas, L.T. Romano, Phase separation in InGaN thick films and formation of InGaN/GaN double heterostructures in the entire alloy composition, Appl. Phys. Lett. 70 (1997) 1089-1091.

[5] J. Ju, B. Loitsch, T. Stettner, F. Schuster, M. Stutzmann, G. Koblmüller, Trade-off between morphology, extended defects, and compositional fluctuation induced carrier localization in high In-content InGaN films, J. Appl. Phys. 116 (2014) 053501.

[6] H. Komaki, R. Katayama, K. Onabe, M. Ozeki, T. Ikari, Nitrogen supply rate dependence of InGaN growth properties, by RF-MBE, J. Cryst. Growth 305 (2007) 12-18.

[7] Ž. Gačević, V.J. Gómez, N.G. Lepetit, P.E.D. Soto Rodríguez, A. Bengoechea, S. Fernández-Garrido, R. Nötzel, E. Calleja, A comprehensive diagram to grow (0001)InGaN alloys by molecular beam epitaxy, J. Cryst. Growth 364 (2013) 123-127.

[8] A. Kraus, S. Hammadi, J. Hisek, R. Buß, H. Jönen, H. Bremers, U. Rossow, E. Sakalauskas, R. Goldhahn, A. Hangleiter, Growth and characterization of InGaN by RF-MBE, J. Cryst. Growth 323 (2011) 72-75.

[9] H. Komaki, T. Nakamura, R. Katayama, K. Onabe, M. Ozeki, T. Ikari, Growth of In-rich InGaN films on sapphire via GaN layer by RF-MBE, J. Cryst. Growth 301-302 (2007) 473-477.

[10] C. Song-Bek, S. Takuro, M. Tomoyasu, W. Xinqiang, I. Yoshihiro, Y. Akihiko, Effect of Precise Control of V/III Ratio on In-Rich InGaN Epitaxial Growth, Jpn. J. Appl. Phys. 45 (2006) L1259.

[11] T. Yamaguchi, N. Uematsu, T. Araki, T. Honda, E. Yoon, Y. Nanishi, Growth of thick InGaN films with entire alloy composition using droplet elimination by radical-beam irradiation, J. Cryst. Growth 377 (2013) 123-126.

[12] M. Moseley, J. Lowder, D. Billingsley, W.A. Doolittle, Control of surface adatom kinetics for the growth of high-indium content InGaN throughout the miscibility gap, Appl. Phys. Lett. 97 (2010) 191902.

[13] M. Moseley, B. Gunning, J. Greenlee, J. Lowder, G. Namkoong, W.A. Doolittle, Observation and control of the surface kinetics of InGaN for the elimination of phase separation, J. Appl. Phys. 112 (2012) 014909.

[14] A.M. Fischer, Y.O. Wei, F.A. Ponce, M. Moseley, B. Gunning, W.A. Doolittle, Highly luminescent, highindium-content InGaN film with uniform composition and full misfit-strain relaxation, Appl. Phys. Lett. 103 (2013) 131101.

[15] E. Iliopoulos, A. Georgakilas, E. Dimakis, A. Adikimenakis, K. Tsagaraki, M. Androulidaki, N.T. Pelekanos, InGaN(0001) alloys grown in the entire composition range by plasma assisted molecular beam epitaxy, Phys. Status Solidi A 203 (2006) 102-105. 